\title{
Synthesis and application of layered titanates in the photocatalytic degradation of phenol
}

Svetlana Ivanova*,Anna Penkova, María del Carmen Hidalgo, José Antonio Navío, Francisca Romero-Sarria, Miguel Ángel Centeno, José Antonio Odriozola

Departamento de Química Inorgánica e Instituto de Ciencia de Materiales de Sevilla (ICMS), Centro mixto CSIC-Universidad de Sevilla, Avda. Americo Vespucio 49, 41092 Sevilla, Spain

E-mail: svetlana@icmse.csic.es

\begin{abstract}
This study proposes a direct synthetic route to single titanate sheets through the mild and versatile conditions of the "chimie douce". The stages of the production include the complexation of the titanium alkoxide precursor by benzoic acid, the formation of titanium oxo-clusters and their controlled transformation into single sheet titanates during the hydrolysis stage. The resulted material appears to be an excellent precursor for self -organized $\mathrm{TiO}_{2}$ nanotubes formation which presents an excellent activity as photocatalyst in the photo-degradation of phenol.
\end{abstract}

Keywords: titanates, self organized $\mathrm{TiO}_{2}$ nanotube layers, photocatalytic degradation of phenol 


\section{Introduction}

Over the past decades, the $\mathrm{TiO}_{2}$ derived nanosized materials have been widely investigated for vast applications, including gas sensors, photocatalysts, solar cells and biomedical applications [1-5]. More precisely, the $\mathrm{TiO}_{2}$ based nanotubes (TNT) have been extensively studied since their peculiar properties, like high specific area, ionexchange ability and photocatalytic activity [6]. Currently developed methods for TNT production include the assisted-template method [7,8], the electrochemical anodic oxidation $[9,10]$ and hydrothermal treatment [11-14]. However, all these methods suffer some drawbacks, like complicated fabrication process in the case of template method, limitation of mass production and high fabrication costs for the electrochemical method and long reaction duration and difficulties in achieving the uniform size nanotubes through the hydrothermal treatment. Nowadays, the most popular method remains the hydrothermal treatment of $\mathrm{TiO}_{2}$ anatase in $\mathrm{NaOH}$ solutions, firstly reported by Kasuga et al. [11].

The layered titanates have recently received a great attention due to their interesting interlayer chemistry, high ability to ion exchange/intercalation reactions and potential applications in the synthesis of new nanomaterials. Moreover, they can undergo exfoliation/delamination upon intercalation of the bulky organic molecules, producing single sheets with distinctive 2D morphology, which are very attractive building blocks for producing artificial architectures, hybrid multilayers or microporous materials [15].

The only known method for the synthesis of layered titanates is the solid-solid reaction at high temperature between $\mathrm{TiO}_{2}$ and an alkali metal carbonate, followed by acid exchange reaction to produce the protonated form $\mathrm{H}_{\mathrm{x}} \mathrm{Ti}_{2-\mathrm{x} / 4} \square_{\mathrm{x} / 4} \mathrm{O}_{4} \cdot \mathrm{H}_{2} \mathrm{O}[16,17]$. 
On the other hand, heterogeneous photocatalysis has found increasing interest as a promising technique for water and air remediation being an environmental friendly and sustainable technology [18]. So far, $\mathrm{TiO}_{2}$ in its form anatase or anatase-rutile mixtures has been the most widely studied material to be used as photocatalyst. However, this material still presents some drawbacks, such as the high degree of recombination of photogenerated charges which limit the efficiency of the photocatalytic processes. Thus, one interesting challenge nowadays is the design and development of tailored nanostructured materials to overcome these limitations. In this context, $\mathrm{TiO}_{2}$ nanotubes due to their $1 \mathrm{D}$ geometry may allow a faster and shorter charge transport to the surface which would lead to less recombination rate and consequently to the improving in the overall efficiency of the material [19].

This study proposes a direct synthetic route to exfoliated layered titanates and their conversion into self-organized $\mathrm{TiO}_{2}$ nanotubes either by a simple contact with sodium hydroxide solution or by a hydrothermal synthesis. The photocatalytic activity of the produced materials is tested by following the photocatalytic degradation of phenol, chosen as model reaction.

\section{Experimental}

\section{Modified sol-gel synthesis}

In a typical synthesis, $1 \mathrm{~mol}$ of titanium (IV) butoxide (Sigma-Aldrich ASC 97\%), and 4 mol of benzoic acid (Sigma-Aldrich ASC 99.5\%), were added to 5 mol of absolute ethanol (Prolabo, 100\%) and stirred under heating up to $92^{\circ} \mathrm{C}$ until the homogenization of the solution. After that, $8 \mathrm{~mol}$ of distilled water for the hydrolysis step was added dropwise and maintain under vigorous stirring for $3 \mathrm{~h}$. The obtained slurry was dried overnight at $80^{\circ} \mathrm{C}$. The excess of organic compounds was removed 
from the resulting solid by Soxlet extraction during $24 \mathrm{~h}$ using ethanol as solvent. The final product was then dried at room temperature. The sample will be labelled as LT.

A portion of the resulting solids was immersed in a $0.1 \mathrm{~mol} \mathrm{~L}^{-1} \mathrm{HCl}$ solution for 24 hours in order to assure the protonic exchange, washed abundantly with distilled water to neutral $\mathrm{pH}$ and finally dried at room temperature. The sample is labelled as $\mathrm{H}-$ LT.

\section{Ti-nanotubes synthesis}

The product, obtained after the modified sol-gel method (LT), was dispersed in an aqueous solution of $\mathrm{NaOH}\left(7 \mathrm{~mol} \mathrm{~L}^{-1}\right)$ and stirred for 24 hours, washed with distilled water and dried at $80^{\circ} \mathrm{C}$. The as prepared sample is called Na-TNT (S).

In a similar way, suspension of the same sol gel product (LT) and $7 \mathrm{~mol} \mathrm{~L}^{-1}$ $\mathrm{NaOH}$ was submitted to autogeneous pressure at $130^{\circ} \mathrm{C}$ for $24 \mathrm{~h}$, and after, washed with distilled water and dried at $80^{\circ} \mathrm{C}$. The as prepared sample is called Na-TNT (HT).

Finally Na-TNT(S) and Na-TNT(HT) solids were immersed in a $0.1 \mathrm{~mol} \mathrm{~L}^{-1} \mathrm{HCl}$ solution for 24 hours, washed abundantly to neutral $\mathrm{pH}$ and finally dried at room temperature. The samples produced are labelled respectively H-TNT (S) and H-TNT (HT).

\section{Characterizations}

X-ray diffraction (XRD) analysis was performed on an X'Pert Pro PANalytical. Diffraction patterns were recorded with $\mathrm{Cu} \mathrm{K} \alpha$ radiation $(40 \mathrm{~mA}, 45 \mathrm{kV})$ over a $2 \Theta-$ range of 3 to $60^{\circ}$ and a position-sensitive detector using a step size of $0.05^{\circ}$ and a step time of $80 \mathrm{~s}$. 
X-ray diffraction (XRD) analysis at high temperature was performed in a high temperature camera Anton Paar HTK 1200 coupled with an X'Pert Pro Philips diffractometer, equipped with $\mathrm{X}^{\prime}$ Celerator detector with an opening of $2.18^{\circ}$, a step of $0.05^{\circ}$ and an equivalent time acquisition of $30 \mathrm{~s}$. The diffractograms were taken every $50{ }^{\circ} \mathrm{C}$ in the 50 to $600^{\circ} \mathrm{C}$ temperature range, over a $2 \theta$-range of 3 to $60^{\circ}$ in flow of synthetic air.

The Raman spectra were recorded on a dispersive Horiba Jobin Yvon LabRam HR800 microscope with a $20 \mathrm{~mW}$ He-Ne green laser $(532.1 \mathrm{~nm})$, without filter and with a $600 \mathrm{~g} \mathrm{~mm}^{-1}$ grating. The microscope used a 50x objective with a confocal pinhole of $1000 \mu \mathrm{m}$.

BET surface area and porosity measurements were carried out by $\mathrm{N}_{2}$ adsorption at $77 \mathrm{~K}$ using a Micromeritics ASAP 2010 instrument.

Transmission electron microscopy (TEM) observations were carried out in a Hitachi H 800 microscope operating at $200 \mathrm{kV}$. The samples were dispersed in ethanol by sonication and dropped on a copper grid coated with a carbon film.

\section{Photocatalytic experiments}

The evaluation of the photocatalytic activity was performed by using the photooxidation of phenol as model reaction.

Suspensions of the samples $\left(1 \mathrm{~g} \cdot \mathrm{L}^{-1}\right)$ in phenol solution $(25 \mathrm{ppm})$ were placed in a $200 \mathrm{ml}$ pyrex discontinuous batch reactor enveloped by an aluminum foil and illuminated through a UV-transparent Plexiglas ${ }^{\circledR}$ top window (threshold absorption at $250 \mathrm{~nm})$ by an Osram Ultra-Vitalux lamp (300 W) with sun-like radiation spectrum and a main line in the UVA range at $365 \mathrm{~nm}$. The intensity of the incident UVA light on the 
solution was determined with a PMA 2200 UVA photometer (Solar Light Co.) being ca. $140 \mathrm{~W} \cdot \mathrm{m}^{2}$.

Magnetic stirring and a constant oxygen flow as oxidative agent were used to produce a homogeneous suspension of the catalyst in the solution. Prior illumination, catalyst-substrate equilibration was ensured by stirring the suspension 20 minutes in the dark. Phenol concentration was followed by HPLC technique (Agilent Technologies 1200) equipped with UV-Vis detector using Elipse XDB-C18 column (5 $\mu \mathrm{m}, 4.6$ x 150 $\mathrm{mm})$. Mobile phase was water/methanol $(65: 35)$ at a flow rate of $0.8 \mathrm{ml} . \mathrm{min}^{-1}$.

Blank experiments were performed in the dark as well as with illumination and no catalyst, without observable change in the initial concentration of phenol in both cases.

\section{Results and discussion}

Before starting the discussion of the results, one precision should be made. In general, the subsequent treatment of all the samples by acid solutions does not provoke any changes in morphology, shape and/or crystallinity. That is why only the characterization of the protonated forms is presented through the whole manuscript. The only difference results in the specific surface area and pore volume as presented in Table 1.

The results of the specific surface area measurements show that the treatment of the sol-gel product sample (LT) with sodium hydroxide solution does not change excessively the initial surface area. However, the subsequent acid treatment and more precisely the abundant washing till neutral $\mathrm{pH}$ provoke a significant increase of the surface area caused by pore liberation. Nevertheless the applied Soxlet extraction in ethanol of the organic phase, the presence of Na-benzoates and/or benzoic acid rests could be imagined as responsible for pore filling into the non-acid treated samples. The 
contact with acid solutions produce mesoporous materials with rather high specific surface area neighbouring $200 \mathrm{~m}^{2} \mathrm{~g}^{-1}$.

The XRD pattern of the synthesized protonated sol-gel product $(\mathrm{H}-\mathrm{LT})$ is presented in Figure 1.

The obtained reflections indicate the production of lamellar structure which can be indexed as $(0 \mathrm{k} 0)$ body centered orthorhombic titanate lepidocrocite like structure. In addition, the asymmetric line shape with a tail toward higher angle diffractions, in the 010 reflection peak, was reported by Sasaki et al. [20] as typical of a two-dimensional lattice, pointing to the production of $2 \mathrm{D}$ network of the layered titanates.

A more detailed study of the H-LT pattern indicates the presence of an amorphous phase, which could be interpreted either as a combination of scattering between water and/or organic molecules (benzoates) in between the titanate sheets or by the presence of low crystalline titania anatase phase which major diffraction appears at $2 \theta \sim 25$ (indicated with the cross symbol in Figure 1). Furthermore, the diffraction lines at around $2 \theta \sim 8$ and $17^{\circ}$ (triangles) specified also the presence of an additional phase identified as crystalline benzoic acid, suggesting that some organic leftovers (more likely benzoates) still exist after the Soxlet extraction and that the acid treatment of the LT sample results, among others, in the conversion of the benzoates to benzoic acid and its crystallization.

On the basis of XRD data, the interlayer distance of $1.6 \mathrm{~nm}$ is measured. This value is significantly higher than the value of $0.93 \mathrm{~nm}$ previously reported for protonated titanates [21], indicating the benzoic acid intercalation between the titanate layers. Using the model proposed by Sasaki et al. [20], and taking into account the size of the benzoic acid molecule, the calculated distance between the titania sheets ranges between 1.3 and $1.8 \mathrm{~nm}$ depending on the presence or absence of water molecules 
intercalation. It is important to mention, that the presence of large excess of benzoic acid, as in our case, suggests strongly an aggregation of the aromatic rings through $\pi-\pi$ stacking, which is also promoted by the presence of functional groups, like carboxylic one. This phenomenon could explain the increase of the interlayer distance, hosting more than one layer of benzoic acid. The described above suggests at first place the presence of benzoic acid and also confirms the stabilization of the 2D lattice through the interlayer benzoic acid/esters intercalation (Figure 2).

As for the TNT samples, both patterns feature the XR Diffraction peak at $2 \theta \sim$ 10, which is a diagnostic for the gallery spacing of the layered titanates (Figure 3 ). The diffractions for the both samples can be assigned as reflections of the basal inter-layer (0k0) series and in-layer (200) of the lepidocrocite type titanate [22, 23]. The crystal structure of titania nanotubes is still under debate, although most studies agreed on the chemical composition $\left(\mathrm{Na}_{\mathrm{x}} \mathrm{H}_{2-\mathrm{x}} \mathrm{Ti}_{3} \mathrm{O}_{7}\right.$ and $\mathrm{Na}_{\mathrm{x}} \mathrm{H}_{2-\mathrm{x}} \mathrm{Ti}_{2} \mathrm{O}_{4}(\mathrm{OH})$ ) attributing the titania nanotubes to the layered titanates group [6].

The sample prepared by hydrothermal treatment (H-TNT (HT)) appears to present higher crystallinity than the corresponding sample prepared by stirring $(\mathrm{H}-$ $\mathrm{TNT}(\mathrm{S})$ ) regarding to the sharpness of the diffraction peaks. For all protonated samples, a XRD study as a function of the temperature in the presence of static air was carried out in order to follow the modification and the phase transitions. As expected, the layered titanates (H-LT) converts rapidly at temperature as low as $200{ }^{\circ} \mathrm{C}$ to the thermodynamically stable anatase form of titania and maintains such phase in the whole range of studied temperatures (up to $600{ }^{\circ} \mathrm{C}$, diffractograms not shown). However, more significant changes are observed in the nanotubes presenting samples. The results are presented in Figure 4 and Figure 5 for H-TNT(S) and H-TNT(HT), respectively. 
The diffractograms of the sample obtained by stirring, H-TNT(S), together with the presence of low crystalline TNT, show the existence of a phase similar to the $\mathrm{NaTiO}_{3}$ one (JCPDS 00-033-1295). It should be mentioned however, that its protonated analogue has not been found in the database, but should give a similar pattern as the sodium form. This titanate phase becomes the major one at higher temperatures, indicating the conversion of the non-stacked lamellar sheets into titanate. The most important result is the heterogeneity of this sample composed by a mixture of TNT and free titanate nanosheets which have been unable to restack incorporating the $\mathrm{Na}^{+}$, in the condition of simple stirring. In addition, the anatase phase (JCPDS 00-021-1272) was observed at temperatures higher than $500{ }^{\circ} \mathrm{C}$. The diffraction peak at around $2 \theta 49^{\circ}$, attributed to pure TNT, does not show any change till the final temperature of the treatment, when disappears. However, the diffraction peak at around $2 \theta \sim 10^{\circ}$ shifts to highest values.

The nanotubes obtained hydrothermally, H-TNT(HT), do not present significant phase transition in the whole range of studied temperatures. However, as for the H-TNT (S), the diffraction peak at around $2 \theta \sim 10^{\circ}$ shifts to higher values, suggesting the shortening of the inter-sheet spacing. This can be due, either to the continuous restacking of the titanate nanosheets, or more likely to the loss of the inter-sheet water content [24], as the change occurs at low temperature (below $150^{\circ} \mathrm{C}$ ).

The laminar structures (LT sample) are clearly visible by transmission electron microscopy (Figure 6 A,) and allow the measure of the interlayer distance, found to be between 1.5 and $3 \mathrm{~nm}$. The distance of $3 \mathrm{~nm}$ suggests that the sample consists of individual nanosheets with no interaction between them, which confirms the ability of the modified sol-gel method to produce single titanate sheets. 
The contact of the single titanate sheets under hydrothermal condition with the sodium hydroxide solution results in a needle shaped 1D structure, having a fibre/tube organization (Figure $6 \mathrm{~B}$ ). In the case of the H- TNT (S) sample, besides the formation of the needle shaped nanotubes (Figure 6C), some less-organized agglomerates of titanates are visible (Figure 6D). The lower extent of organization of this type of samples was already described in the literature, and an improvement of the sample morphology could be substantially obtained by heating or by repeating cycles with fresh $\mathrm{NaOH}$ solutions $[22,25]$. The hydrothermal treatment results in more complete transformation of the single titanate sheets to nanotubes, as confirmed by the XRD and TEM.

The Raman scattering spectrum of the protonated forms of the initial layered titanate sample and of the self-organized nanotubes structures are presented in Figure 7. In a detailed study of the protonated titanates with lepidocrocite type layered structures, Gao et al. [15] observe nine Raman modes as predicted by the Group theory $\left(3 \mathrm{~A}_{\mathrm{g}}+\right.$ $3 \mathrm{~B}_{1 \mathrm{~g}}+3 \mathrm{~B}_{3 \mathrm{~g}}$ ) at the $100-1000 \mathrm{~cm}^{-1}$ spectral region attributed to the Ti--O lattice vibrations within the $2 \mathrm{D}$ lepidocrocite-type $\mathrm{TiO}_{6}$ octahedral host layers. They recorded the lattice vibration at $183,270,387,449,558,658,704,803$ and $908 \mathrm{~cm}^{-1}$. Three of them (the symmetric $\mathrm{A}_{\mathrm{g}}$ modes) are recognized as indicative of a well-developed 2D layered structure, namely $270,449,704 \mathrm{~cm}^{-1}$. All of them are present in the selforganized nanotubes samples produced in this study. The presence of those three bands represents the clear signature used for in situ probing of 2D titanate nanosheets and their derivatives, as nanotubes $[26,27]$. The number of observed modes in this study agrees fairly well with the space group theoretical analysis of lepidocrocite type titanate structure, thus confirming the production of the TNT of $\mathrm{H}_{\mathrm{x}} \mathrm{Ti}_{2-\mathrm{x} / 4} \square_{\mathrm{x} / 4} \mathrm{O}_{4} \cdot \mathrm{H}_{2} \mathrm{O}$ form. 
Nonetheless the clarity of the assignment of the nanotube samples to the group of the lepidocrocite type titanates, the Raman spectra is not so conclusive in the case of the initial layered structure. Although some similarity with the nanotube samples exists some additional vibration bands could be observed, e.g. at around 330, 620 and especially, the sharp band at $847 \mathrm{~cm}^{-1}$. This band was reported by Kudo et al. [28] for the layered $\mathrm{Cs}_{2} \mathrm{Ti}_{n} \mathrm{O}_{2 n+1}(\mathrm{n}=2,5)$ with layered structure consisting of $\mathrm{TiO}_{5}$ units. This observation suggests that, probably, two types of $\mathrm{Ti}$ oxo-clusters are formed in the presence of benzoic acid leading to two types of layered titanates, built either by $\mathrm{TiO}_{5}$ or $\mathrm{TiO}_{6}$ units. During the sol gel reaction the production of carboxylate-substituted polyalkoxy clusters with varied nuclearity via dynamic alcohol exchanging reactions and esterification is expected. The condensation degree of the clusters depends on the complexing ratio ([L]/[Ti]), where $\mathrm{L}$ is benzoic acid concentration and on the hydrolysis ratio $([\mathrm{H} 2 \mathrm{O}] /[\mathrm{Ti}])[29]$. The ratios used in this study suggests low nuclearity clusters formation, as for example $\mathrm{Ti}_{3} \mathrm{O}(\mathrm{OR})_{8}(\mathrm{OOCPh})_{2}$ and/or $\mathrm{Ti}_{4} \mathrm{O}_{2}(\mathrm{OR})_{10}(\mathrm{OOCPh})_{2}$, where $\mathrm{R}$ could be ethyl or butyl groups. The entering of one benzoate ligand into Ti coordination sphere proceeds via two OR groups replacement leading to the $\mathrm{Ti}$-coordination number decrease and readjusting in the coordination mode of all the other ligands, thus producing the titanate like laminar arrangements [30]. This phenomenon, also called "poisoning" effect towards oxo-polymers growth was observed by Roses et al. [31] when strong complexating agent such as benzoic acid is used.

\section{Photocatalytic activity}

The evaluation of the photocatalytic activity was carried out by following the reaction of oxidation of phenol and degradation profiles over the different samples are 
shown in Figure 8. In all cases a total mineralization occurs and no products of selective oxidation are detected.

A clear difference between the sodium containing materials and their protonated homologues can be seen. Whereas the presence of sodium decreases strongly the rate of the oxidation, the presence of $\mathrm{H}^{+}$increases the reaction rate noticeably. This effect could be explained regarding the mechanism of degradation of organic pollutants. It was reported [32] that the primary oxidant in this type of reaction is $\mathrm{OH}^{-}$, generated from the oxidation of adsorbed water, and that the presence of oxygen prevents the recombination of an electron - hole pair. The use of protonated forms of the layered titanates and self-organized nanotubes structures increases the density of the surface hydroxyl groups, thus promoting the activity.

Moreover, the presence of sodium on $\mathrm{TiO}_{2}$ photocatalysts has been proven to be detrimental for its photocatalytic activity as these ions may act as recombination centres for the photogenerated charges thus reducing the efficiency of the photocatalytic process [33-35]. Therefore, a high degree of recombination in the nanotubes before their treatment in acid solutions can be expected, inhibiting the photocatalytic activity of these samples, as it can be clearly observed in Figure 8 .

The protonic exchange results in mesoporous materials with higher surface area than that of the Na analogues, which also helps to increase the activity. However, in the case of the protonated samples, this increase in activity cannot be ascribed to the higher surface area as it can be seen in Figure 9 where the rate of phenol degradation per surface area unit of photocatalyst is presented.

When activity per surface area is considered, the protonated nanotube materials still show much higher activity than their $\mathrm{Na}$ containing counterparts. However, the 
nanotubes prepared by simple stirring and those prepared by hydrothermal method present a similar activity per surface area unit of the photocatalyst.

Despite of the extensive study of the nanotube based materials in lithium ion batteries, electrochemical devices, gas sensors, photoluminiscense ion exchange and photovoltaic dye sensitized solar cells [36, 37], its photocatalytic ability is majorly judged as insufficient. The TNT materials, although in close partnership with anatase the phase of $\mathrm{TiO}_{2}$, do not inherit its photocatalytic ability at least without thermal treatment [38]. However it can be found in the literature few studies showing the higher efficiency of the TNT materials compared to anatase $\mathrm{TiO}_{2}[37,39]$, ascribed to the more optimized geometry of the nanotubes and to the shorter carrier - diffusion path in the tube walls including lower trapping and recombination kinetics of light-generated electron-hole pairs compared to standard $\mathrm{TiO}_{2}$ when the pairs have to travel in between the single nanoparticles. It is important to mention that the exfoliated titanate sample (H-LT) shows a higher efficiency in phenol degradation than the pure TNT materials reported by Hussain et al. [37]. This activity could be attributed either to the layered titanates itself or to the help provided by the presence of the anatase phase, as detected by XRD. The presence of the benzoic acid traces however does not influence the catalytic results as seen by the second run performed for this sample. The resulting selforganized TNT structures from the LT sample present higher efficiency and, which is the more interesting, the material prepared by simple contact with sodium hydroxide solution shows the highest activity reaching the total elimination of phenol within the 2 $\mathrm{h}$ of the test. The presence of a mixture of nanotubes and less organized titanates, as seen by TEM, seems to be beneficial for the photocatalytic activity, suggesting that the time consuming and expensive hydrothermal treatment is no more needed. 
In summary, as reported in the literature [22, 23], the formation of the nanotubes from titanates always goes through the previous exfoliation step, which could be a very time consuming step; from two days for the lepidocrocite type titanates to two weeks for a certain type of titanates. In addition, the use of quaternary ammonium salts for this process appears to be an expensive step, together with the titanates production via high temperature solid-solid reaction.

According to our results, the modification of the process of formation of the titania precursor during the sol-gel process by benzoic acid results in simpler direct arrangements of the titanium oxo-clusters before the hydrolysis and condensation step into single titanate sheets. These valuable 2D building blocks can generate $1 \mathrm{D}$ structures with nanotube self-organization by a simple contact with concentrated $\mathrm{NaOH}$ solution even at room temperature following the mechanism proposed by Ma et al. [40]. However, the most appropriate method to produce a homogeneous, highly crystalline and pure TNT material seems to be the hydrothermal method. Nevertheless, with the suitable sodium hydroxide treatment conditions, we do believe that the easily produced sol-gel single titanate sheets could be converted into one unique highly crystalline titanium nanotubes phase. Regarding the photocatalytic results, it appears that certain heterogeneity of the sample is beneficial for the catalytic performance, at least in the photodegradation of phenol.

\section{Conclusions}

This study shows that the utilisation of the sol-gel method with suitable modificator could result in an easier and cost saving method for single titanate sheets preparation. 
The single titanate sheets could form titania nanotubes directly by a simple contact with strongly basic solutions of $\mathrm{NaOH}$ without any additional treatment, as heating or long duration treatment. However, the as prepared nanotubes show lower crystallinity and homogeneity than the hydrothermal prepared samples.

Nevertheless, this partial heterogeneity results beneficial from photocatalytic point of view, presenting a co-catalyst effect when nanotubes and layered titanates coexist, indicating the versatility of the synthesized material.

\section{Acknowledgements}

Financial support for this work has been obtained from Junta de Andalucía (Plan Andaluz de Investigación, grupo TEP106) and the Spanish Ministerio de Ciencia e Innovación (CTQ2011-26617-C03-02). S. Ivanova, F. Romero-Sarria and A. Penkova acknowledge MEC for their contract Ramón y Cajal and Juan de la Cierva. CITIUS is kindly acknowledged to provide the TEM for all the measurements in this study.

\section{References}

[1] M. Gratzel, Nature, 414 (2001) 338-344.

[2] U. Bach, D. Lupo, P. Comte, J. E. Moser, F. Weissortel, J. Salbeck, H. Spereitzer, M.Gratzel, Nature, 395 (1998) 583-585.

[3] T. Lopez, S. Recillas, P. Guevara, J. Sotelo, M. Alvarez, J. A. Odriozola, Acta Biomat.,4 (2008) 2037-2044.

[4] M. I. Litter, J. A. Navio, J. Photochem. Photobiol. A: Chem., 91 (1996) 171-181.

[5] V. F. Stone Jr., R. J. Davis, Chem. Mater., 10 (1998) 1468-1474.

[6] H.-H. Ou, S.-L. Lo, Separ. and Purif. Techn., 58 (2007)179-191. 
[7] J.H. Jung, H. Kobayashi, K.J.C. van Bommel, S. Shinkai, T. Shimizu, Chem. Mater., 14 (2002) 1445-1447.

[8] J. H. Lee, I. C. Leu, M. C. Hsu, Y. W. Chung, M. H. Hon, J. Phys. Chem. B, 109 (2005) 13056-13059.

[9] D. Gong, C.A. Grimes, O.K. Varghese, W. Hu, R.S. Singh, Z. Chen, E.C. Dickey, J. Mater. Res., 16 (2001) 3331-3334.

[10] A. Ghicov, H. Tsuchiya, J.M. Macak, P. Schmuki, Electrochem. Commun., 7 (2005) 505-507.

[11] T. Kasuga, M. Hiramatsu, A. Hoson, T. Sekino, K. Niihara, Adv. Mater., 11 (1999) $1307-1311$.

[12] G.H. Du, Q. Chen, R.C. Che, Z.Y. Yuan, L.M. Peng, Appl. Phys. Lett., 79 (2001) $3702-3704$.

[13] Q. Chen,W.Z. Zhou, G.H. Du, L.M. Peng, Adv. Mater., 14 (2002)1208-1211.

[14] Q. Chen, G.H. Du, S. Zhang, L.M. Peng, Acta Cryst. B, 58 (2002) 587-593.

[15] T. Gao, H. Fjellvag, P. Norby, J. Phys. Chem. B, 112 (2008) 9400-9405.

[16] T. Sasaki, M. Watanabe, Y. Michiue, Y. Komatsu, F. Izumi, S. Takenouchi, Chem. Mater., 7 (1995) 1001-1007.

[17] T. Sasaki, F. Kooli, M. Iida, Y. Michiue, S. Takenouchi, Y. Yajima, F. Izumi, B. C. Chakoumakos, M. Watanabe, Chem. Mater., 10 (1998) 4123-4128.

[18] D. Friedmann, C. Mendive, D. Bahnemann, Appl. Catal. B: Environ., 99 (2010) 398-403.

[19] T. Tachikawa, S. Tojo, M. Fujitsuka, T. Sekino, T. Majima, J. Phys. Chem. B 110 (2006) 14055-14059.

[20] T. Sasaki, M. Watanabe, H. Hashizume, H. Yamada, H. Nakazawa, J. Am. Chem. Soc. 118 (1996) 8329-8335. 
[21] T. Sasaki, M. Watanabe, J. Am. Chem. Soc., 120 (1998) 4682-4689.

[22] T. Kasuga, M. Hiramatsu, A. Hoson, T. Sekino, K. Niihara, Adv. Mater., 11 (1999) $1307-1311$.

[23] T. Sasaki, M. Watanabe, J. Am. Chem. Soc., 120 (1998) 4682-4689.

[24] R. Ma, Y. Bando, T. Sasaki, J PhysChem B, 108 (2004) 2115-2119.

[25] R. Ma, K. Fukuda, T. Sasaki, M. Osada, Y. Bando, J Phys Chem B, 109 (2005) 6210-6214.

[26] D. V. Bavykin, J. M. Friedrich, A. A. Lapkin, F. C. Walsh, Chem. Mater., 18 (2006) 1124-1129.

[27] T. Sasaki, M. Watanabe, J. Chem. Phys. B., 101 (1997) 10159.

[28] A. Kudo, T. Kondo, J. Mater. Chem., 7 (1997) 777-780.

[29] C. Sanchez ,F. Ribot, New J Chem, 18 (1994) 1007-1047.

[30] I. Mijatovic, G. Kickelbick, U. Schubert, Eur. J. Inorg. Chem. (2001) 1933-1935.

[31] L. Rozes, N. Steunou, G. Fornasieri, C. Sanchez, Monatshefte fur Chemie, 137 (2006) 501-528.

[32] S. Ahmed, M.G. Rasul, W.N. Martens, R. Brown, M.A. Hashib, Desalination, 261 (2010) 3-18.

[33] H. Yu, J. Yu, B. Cheng, Catal. Commun., 7 (2006) 1000-1004.

[34] J-M. Herrmann, H. Tahiri, C. Guillard, P. Pichat, Catal. Tod., 54 (1999) 131-141

[35] Y. Paz, A. Heller, J. Mater. Res., 12 (1997) 2759-2766.

[36] D. Kuang, J. Brillet, P. Chen, M. Takata, S. Uchida, H. Miura, ACS Nano, 2 (2008) $1113-1116$.

[37] S. T. Hussain, A. Siddiqa, Int. J. Environ. Sci. Tech., 8 (2011) 351-362.

[38] H.-H. Ou, Sh. - L. Lo Separation and Purification Technology, 58 (2007) 179-191.

[39] J. M. Macak, M. Zlamal, J. Krysa, P. Schmuki, Small, 3 (2007) 300-304. 
[40] R. Ma, Y. Bando, T. Sasaki, Chem. Phys. Lett., 380 (2003) 577-582. 